\title{
Inverse Kinematics of Redundant Manipulator using Interval Newton Method
}

\author{
Virendra Kumar ${ }^{a}$, Soumen Sen ${ }^{\mathrm{a}}$, S.S. Roy ${ }^{\mathrm{b}}$, S.K Das ${ }^{\mathrm{a}}$, S.N.Shome ${ }^{\mathrm{a}}$ \\ ${ }^{a}$ Robotics \& Automation Division, CSIR-Central Mechanical Engineering Research Institute, Durgapur, \\ 713209 India \\ ${ }^{b}$ Mechanical Engineering Department, National Institute of Technology, Durgapur, 713209 India
}

\begin{abstract}
The paper presents an application of Interval Newton method to solve the inverse kinematics and redundancy resolution of a serial redundant manipulator. Such inverse problems are often encountered when the manipulator link lengths, joint angles and end-effector uncertainty bounds are given, which occurs due to because of inaccuracies in joint angle measurements, manufacturing tolerances, link geometries approximations, etc. The inverse kinematics of three degree of freedom planar redundant positioning manipulator without endeffector has been evaluated using the manipulability of Jacobian matrix as performance metric. To solve the nonlinear equation of inverse kinematics, the multidimensional Newton method is used. The inverse kinematics is intended to produce solutions for joint variables in interval of tolerances for specified end effector accuracy range. As exemplar problem solving, a planar 3-degrees-of-freedom serial link redundant manipulators is considered.
\end{abstract}

Index Terms: Interval Newton Method, Redundant Manipulator, Inverse Kinematics, Manipulability.

(C) 2015 Published by MECS Publisher. Selection and/or peer review under responsibility of the Research Association of Modern Education and Computer Science.

\section{Introduction}

In robotics, a manipulator is called kinematically redundant if the number of degree of freedom (DOF) of the joint space is higher than the number of degree of freedom of task space coordinates. Inverse kinematics redundancy resolution for redundant serial manipulators remains a key topic in many robotics problems, where, presence of additional degrees of freedoms enhances manipulator's ability, and task versatility [1-4]. Secondary task of a redundant manipulator is defined by performance indices. These indices include, among many others, isotropic velocity behaviour, manipulability, dynamic manipulability, etc. Manipulability of the manipulator jacobian matrix is used by Yoshikawa [1] as performance metric for manipulating ability of robotic

* Corresponding author. Tel.: +91 3436452048 ;

E-mail address: vkumar@cmeri.res.in 
mechanisms in positioning and orienting end-effectors. Similarly condition number of the manipulator jacobian matrix is used by Salisbury [3] as an optimization performance criterion to evaluate the dimensions for the fingers of the Stanford/JPL articulated hand. To design a manipulator, Klein [4] used the condition number of jacobian matrix of manipulator for isotropy at a working point for a fixed total arm length. One problem of using condition number of a Jacobian matrix is the mismatch of units of rectilinear and rotational velocities. To overcome this discrepancy a characteristic length of manipulator is proposed by Angeles [5] to normalize the manipulator jacobian.

This article addresses the problem of inverse kinematics evaluation of redundant manipulator using an Interval Arithmetic based technique. This interval based technique is not much explored for redundant serial manipulator problem, can lead to design optimization of manipulators with dimensional/length uncertainties due to manufacturing tolerance, assembly variations and uncertainty inherent in behaviour of procured components, given that the uncertainty ranges are known. Some literatures are available, where, the researchers attempted to solve the inverse kinematics of non-redundant serial manipulator by the interval methods [6-8]. Castellet et al. [7] solved the inverse kinematics by using the n-bar mechanism and form a closed single-loop mechanism and apply the interval Newton cut approach. Roa et. al. [6] applied the interval Krawczyk method to solve the inverse kinematics equation of industrial robots. Pac et. al. [8] used the SIVIA algorithm (Set Inversion via Interval Analysis algorithm) to solved the solution to the inverse kinematics problem of the twolink and three link manipulator. This article attempts to solve the redundancy resolution and inverse kinematics of redundant manipulator by formulating the optimization problem as manipulability of Jacobian should be maximum. The maximization equality problem is converted in the form of General Fritz-John formulation and solved it using Interval Newton method.

The organization of the paper contains the problem formulation for inverse kinematics of redundant manipulator and some basics of interval arithmetic in Section 2. Section 3 describing the procedure for inverse kinematics solution with 3-DOF manipulator example, and conclusions are delineated in Section 4.

\section{Problem Formulation and Interval Method}

\subsection{Inverse Kinematics Problem}

The forward kinematic equation of an $n$-DOF manipulator can be expressed as

$$
x=f(q)
$$

where, $x \in R^{m}$ is the end effector pose in $m$-dimensional task space, $q \in R^{n}$ is the joint space variable vector of dimension $n$ and $f$ is a nonlinear vector function obtained from the particular kinematic structure of the manipulator. Inverse kinematics requires to find $f^{-1}$ in order to find one or more joint angle vectors for a given end-effector position and orientation, such that

$$
q=f^{-1}(x)
$$

Solution of (1), for redundant manipulator, gives an infinite number of configurations. Although in principle (2) may exist, in practice it is not easy to obtain a closed form inverse kinematic function of (1) for spatial manipulators having more than three degrees of freedom in particular. In such a case, iterative and algorithmic techniques are generally employed. One such technique makes use of jacobian of the forward kinematic function in (1), this is defined in the differential motion relations

$$
\dot{x}=J(q) \dot{q} \text { or } \Delta x=J(q) \Delta q
$$


where, $J=\partial f / \partial q_{i}, i=1, . ., n$. Inverse kinematics is solved either using first of (3) and integrating the velocities for position with respect to an initial configuration, or, by an iterative method using increment in second of (3) again with knowledge of an initial configuration. A specific inverse kinematic solution with minimum norm can be obtained by using the Moor-Penrose pseudo inverse [9] of J(q) but it does not guarantee avoidance of all occurrences of singularity. A more general solution of second of (3) is given by

$$
\Delta q=J^{\#} \Delta x+\left(I_{n x n}-J^{\#} J\right) \Delta q_{0}
$$

which is a non-minimum norm solution, where a homogeneous term is added to the minimum norm solution. $I_{n}$ $x_{n}$ is an identity matrix of dimension $n$ and $\Delta q_{0}$ is an arbitrary vector, denoting null-space motion, which is added with the minimum-norm-term through a projection operator which is added with the minimum-normterm through a projection operator $\left(I_{n x n}-J^{\#} J\right)$. This non-minimum norm solution allows reaching a secondary objective and achieves Manipulability. In this article, Manipulability of Jacobian is taken as the performance metric. The redundancy resolution and inverse kinematics is solved by prioritizing the tasks - the primary task being the reachability to the goal point in the workspace and the secondary task is maximize the manipulability of Jacobin matrix. Based on the primary and secondary task of manipulator formulate the optimization problems and solve them using interval Newton method. In this article, the inverse kinematic solution is attempted using optimization problem that is based on manipulability of Jacobian matrix, by incorporating interval technique to handle uncertainties in link dimensions and goal position.

\subsection{Interval Arithmetic}

Interval analysis is a relatively new mathematical branch of computational mathematics where computations are carried out on intervals instead of real numbers. Interval analysis is used to design interval algorithms for solving systems of linear and nonlinear equations and optimization problems [10-12]. Moore [13] among few firsts made important discussions with the basic interval operations.

An interval $\mathrm{X}$ is defined as the closed bounded set of real numbers $x$ denoted by $X=[\underline{X}, \bar{X}]$, such that, $\underline{x} \leq x \leq \bar{x}$ where $\underline{x}, \bar{x}$, represent lower and upper bounds respectively. Four important elementary definitions based on unary operations are stated as:

a Midpoint: $m(X)=(\underline{x}+\bar{x}) / 2$

b Width: $w(X)=(\bar{x}-\underline{x})$

c Radius: $\operatorname{rad}(X)=(\bar{x}-\underline{x}) / 2$

d Absolute value: $|X|=\max \{|\bar{x}|,|\underline{x}|\}$

(Note that $|x| \leq|X|$ for every $x \in X$ )

The basic interval arithmetic operations are defined on interval vector such that the interval result includes all possible real values. For a given interval $X=[\underline{X}, \bar{X}]$ and $Y=[\underline{Y}, \bar{Y}]$ the elementary operations are defined as Xоp $Y=\{$ xopy $: x \in X, y \in Y\}$ where $o p$ denotes one of $\{+,-, \times, \div\}$. In case of division, $0 \notin Y$.

(a) Addition: $X+Y=[\underline{x}+y, \bar{x}+\bar{y}]$

(b) Subtraction: $X-Y=[\underline{x}-\bar{y}, \bar{x}-\underline{y}]$ 
(c) Multiplication: $X \times Y=[\min \{\underline{x} \times \underline{y}, \bar{x} \times \bar{y}, \underline{x} \times \bar{y}, \bar{x} \times \underline{y}\}, \max \{\underline{x} \times \underline{y}, \bar{x} \times \bar{y}, \underline{x} \times \bar{y}, \bar{x} \times \underline{y}\}]$

(d) Division: $X / Y=[\underline{x}, \bar{x}] \times[1 / \bar{y}, 1 / \underline{y}]$ if $0 \notin Y$.

(e) Intersection: $X \cap Y=[\max \{\underline{x}, \underline{y}\}, \min \{, \bar{x}, \bar{y}\}]$

(f) Union: $X \cup Y=\left\{\begin{array}{l}{[\min \{\underline{x}, \underline{y}\}, \max \{, \bar{x}, \bar{y}\}] \text { if } X \cap Y \neq \phi} \\ \text { undefined, otherwise }\end{array}\right.$

An interval function is an interval valued function of one or more variables. For interval $X=(X 1, \ldots \ldots, X n)$, an interval function $F(X 1, \ldots ., X n)$ is said to be an interval extension of a real function $F(x 1, \ldots \ldots, x n)$ if

$$
F(x 1, \ldots \ldots, x n) \in F(X 1, \ldots ., X n)
$$

$x_{i} \in X_{i}$ for all $i=1, \ldots, n$. An interval function $F$, is called inclusion monotonic if $X_{i} \subset Y_{i}$ implies $F(X 1, \ldots ., X n) \subset F(Y 1, \ldots ., Y n)$.

Interval extension of a mathematical function can be derived by partitioning its domain into monotonic regions. For example, the interval extension of $\sin (X)$ for 0 to $2 \pi$ can be formulated as:

$$
\sin (X)=\left\{\begin{array}{l}
{[\sin (\underline{x}), \sin (\bar{x})] \quad\{0 \leq \underline{x} \leq \bar{x} \leq \pi / 2\} \cup\{3 \pi / 2 \leq \underline{x} \leq \bar{x} \leq 2 \pi\}} \\
{[\sin (\bar{x}), \sin (\underline{x})] \quad \pi / 2 \leq x \leq \bar{x} \leq 3 \pi / 2} \\
{[\min (\sin (\underline{x}), \sin (\bar{x})), 1] \quad\{0 \leq \underline{x} \leq \pi / 2\} \cap\{\pi / 2 \leq \bar{x} \leq 3 \pi / 2\}} \\
{[-1, \max (\sin (\underline{x}), \sin (\bar{x}))] \quad\{\pi / 2 \leq \underline{x} \leq 3 \pi / 2\} \cap\{3 \pi / 2 \leq \bar{x} \leq 2 \pi\}} \\
{[-1,1] \quad\{0 \leq \underline{x} \leq \pi / 2\} \cap\{3 \pi / 2 \leq \bar{x} \leq 2 \pi\}}
\end{array}\right.
$$

Uniform subdivision of an interval vector $X=(X 1, \ldots ., X n)$ can be defined as below [13]:

Let $N$ be a positive integer and define

$$
X_{i, j}=\left[\underline{X}_{i}+(j-1) w\left(X_{i}\right) / N, \underline{X}_{i}+j w\left(X_{i}\right) / N\right], \quad j=1,2, \ldots . N .
$$

Equation (6) is used for generating uniform subdivision of the angle vector for the given angle range in the inverse kinematics procedure.

Intervals methods may be used in many ways to solve the nonlinear equations. Mostly these methods can be described in terms of contraction operators, or contractors [13]. Function of the contractors is either reducing the size of, or completely eliminates, the region in which solutions to the equation system of interest are being sought. The contraction strategies based methods are Krawczyk and interval-Newton methods that have been widely used in the solution of nonlinear equation systems. Here we next described the interval-Newton methods only which is used in this paper to solve the nonlinear equations.

\subsubsection{Newton Method}

Let us consider the nonlinear equations $f(x)=0$, such that we need a solution in vector $x$. Suppose that $f(x)$ has a continuous derivative in the region of interest. By the mean value theorem: 


$$
f(x)=f(y)+f^{\prime}(\varepsilon)(x-y)
$$

where $\varepsilon$ lies between $x$ and $y$. Now assume $f(x)=0$, and now (7) became

$$
x=y-f(y) / f^{\prime}(\varepsilon)
$$

Suppose $X=[a, b]$, be an interval containing $x$ and $y$, and $F^{\prime}(X)$ be an inclusion monotonic interval extension of $f^{\prime}(X)$ hence interval Newton algorithm [13] can be written as

$$
X^{(k+1)}=X^{(k)} \cap N\left(X^{(k)}\right) \quad k=0,1,2, \ldots \ldots
$$

where

$$
N(X)=m(X)-f(m(X)) / F^{\prime}(X)
$$

and

$$
m(X)=(a+b) / 2
$$

The above solution is for one dimensional variable. For multivariable case (7) can be written as

$$
f(y)-f(x)=A(y-x)
$$

Where $\mathrm{A}$ is a matrix whose $\mathrm{i}^{\text {th }}$ row is given by

$$
A_{i}=\nabla^{T} f_{i}\left(c_{i}\right)=\left(\frac{\partial f_{i}}{\partial x_{1}}\left(c_{i}\right), \ldots \ldots \ldots \ldots \ldots \ldots . . \frac{\partial f_{i}}{\partial x_{n}}\left(c_{i}\right)\right)
$$

To solve the multidimensional interval Newton method set the equation as assume $f(x)=0$, then

$$
x=y-A^{-1} f(y)
$$

Here A is replaced with $F^{\prime}(X)$. For simplicity it can be written as

$$
x \in N(X)=y+V
$$

Where $V$ bound the solution set to

$$
F^{\prime}(X) v=-F(y)
$$

Using the Interval Gauss' elimination method solve (15). $N(X)$ in (14) is the multidimensional analog to (10). 


\section{Inverse Kinematics for Redundant Manipulator}

Here the inverse kinematic of the manipulator is obtained based on optimization method for the redundancy resolution. The inverse kinematics of the 3-DOF planar manipulator is being studied with maximize the Manipulability of Jacobian as objective function and reachability as constraints.

The considered manipulator configuration of a 3-DOF planar redundant manipulator is shown in Fig. 1, which consists of three links of lengths $l_{1}, l_{2}$ and $l_{3}$. The inverse kinematics of the manipulator for a given task position $\left(x_{g}, y_{g}\right)$ is solved by formulating optimization problem as maximize the manipulability.

The manipulability of a manipulator is defined as

$$
w=\sqrt{\operatorname{det}\left(J J^{T}\right)}
$$

where $J$ is Jacobian of the manipulator.

Thus we formulate the problem as to find the value of angles $\theta_{1}, \theta_{2}$ and $\theta_{3}$ that minimizes

$$
P(\theta)=1 / w
$$

In the bounded region of angles ranges, subject to the $j(j=1,2)$ equality constraints that satisfied the goal position i.e. $g_{j}(\theta)=0$, is given below

Subject to: $x_{g}-\left(l_{1} \cos \left(\theta_{1}\right)+l_{2} \cos \left(\theta_{1}+\theta_{2}\right)+l_{3} \cos \left(\theta_{1}+\theta_{2}+\theta_{3}\right)\right)=0$

$$
x_{g}-\left(l_{1} \sin \left(\theta_{1}\right)+l_{2} \sin \left(\theta_{1}+\theta_{2}\right)+l_{3} \sin \left(\theta_{1}+\theta_{2}+\theta_{3}\right)\right)=0
$$

where $\left(x_{g}, y_{g}\right)$ is the goal position of the manipulator in the workspace.

The equality constraint in (17) and (18) satisfies the reachability criterion (primary task), such that the vector function $g(q)=x-f(q)=0$ gets satisfied for given task position.

General Fritz-John method [11] is applied to solve above equality optimization problem using interval method. The normalized Fritz-John condition for above can be written as below

$$
\begin{aligned}
& u_{0} \nabla P(\theta)+\sum_{j=1}^{2} v_{j} \nabla g_{j}(\theta)=0 \\
& g_{j}(\theta)=0 \\
& u_{0}+\sum_{j=1}^{2} v_{j}^{2}=1
\end{aligned}
$$

The initial bounds for multipliers are $0 \leq u_{0} \leq 1$ and $-1 \leq v_{i} \leq 1$.

The interval Newton method is applied to the Fritz John conditions. The algorithm developed here works on 
boxes, each box being a set of intervals of angles. We assume the initial box $\mathrm{X}(0)$ is given in which the solution is sought. The algorithm is described below as:

\section{Algorithm:}

To solve the (19), (20), and (21) simultaneously $(f(x)=0)$ using interval Newton method the following steps are followed.

(i) Initial interval vector $X(0)$ (i.e., range of angles based on requirement) is given which forms a box.

(ii) Angle ranges are divided into several sub-boxes using the uniform interval subdivision strategy by applying (6) and form Stack, $L_{l}$.

(iii) Take one by one a sub box from Stack $\mathrm{L}_{1}$ and repeat through step (x) until the Stack is empty.

(iv) Take one box $\mathrm{X}$ to be processed, find the value of $F(X)$.

(v) Check, if $0 \notin F(X)$, means it do not have root. go to step (x)

(vi) Using (14) multidimensional interval Newton method, find $N(X)$

$$
\text { if } N(X) \cap X=\phi \text {, then go to step (x) }
$$

(vii) If $N(X) \subseteq X=X$, output $X$ go to step (x)

(viii) Now bisect $X$ such that $X=X^{(1)} \cup X^{(2)}$

$$
\begin{aligned}
& X^{(1)}=[\underline{X},(\underline{X}+\bar{X}) / 2] \\
& X^{(2)}=[(\underline{X}+\bar{X}) / 2, \bar{X}]
\end{aligned}
$$

(ix) Push $X^{1}, X^{(2)}$ into the Stack

(x) $\quad$ Set $X=$ top of Stack

\subsection{Example: 3-DOF Planar Manipulator}

The considered revolute type 3 DOF planar manipulator configuration is shown in Fig. 1, which consists of three links of lengths $l_{1}, l_{2}$ and $l_{3}$. The inverse kinematics of the manipulator for a given task position is solved using the above interval mentioned method. Interval calculations are carried out by using MATLAB and a toolbox called INTLAB developed by S. M. Rump [14].

The considered 3-DOF planar manipulators has a reach of $1.2 \mathrm{~m}$ along with links length as $l_{1}=0.6 \mathrm{~m}$, $l_{2}=0.3 \mathrm{~m}$ and $l_{3}=0.3 \mathrm{~m}$, with manufacturing tolerances $10^{-4} \mathrm{~m}$ and desired goal point is chosen to be $x_{g}=0.8 \mathrm{~m}$, $y_{g}=0.8 \mathrm{~m}$ with precision considered in the range of $\pm 0.001 \mathrm{~m}$. The angle ranges of the joints considered are $q_{1}=-\pi / 2$ to $\pi, q_{2}=-\pi / 2$ to $\pi$ and $q_{3}=-\pi / 2$ to $\pi / 2$.

The link lengths can be represented in interval form as follows:

$$
\begin{aligned}
& l_{1}=[0.5999,0.6001] \\
& l_{2}=[0.2999,0.3001] \\
& l_{3}=[0.2999,0.3001]
\end{aligned}
$$


Similarly the goal point can be represented in interval form as follows

$$
x_{g}=[0.799,0.801], y_{g}=[0.799,0.801]
$$

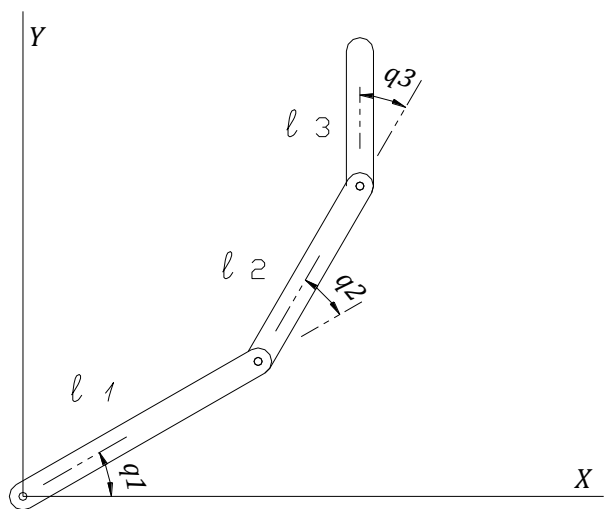

Fig.1. 3-DOF Planar Manipulator

The output angles are as follows

$$
\begin{aligned}
& q 1=[0.4783,0.4791] \\
& q 2=[0.4187,0.4191] \\
& q 3=[0.3989,0.3990]
\end{aligned}
$$

The algorithm output is plotted in Fig. 2, in which the outer box represent the goal position and the inner box represent the manipulator end effector position (manipulator tip position).

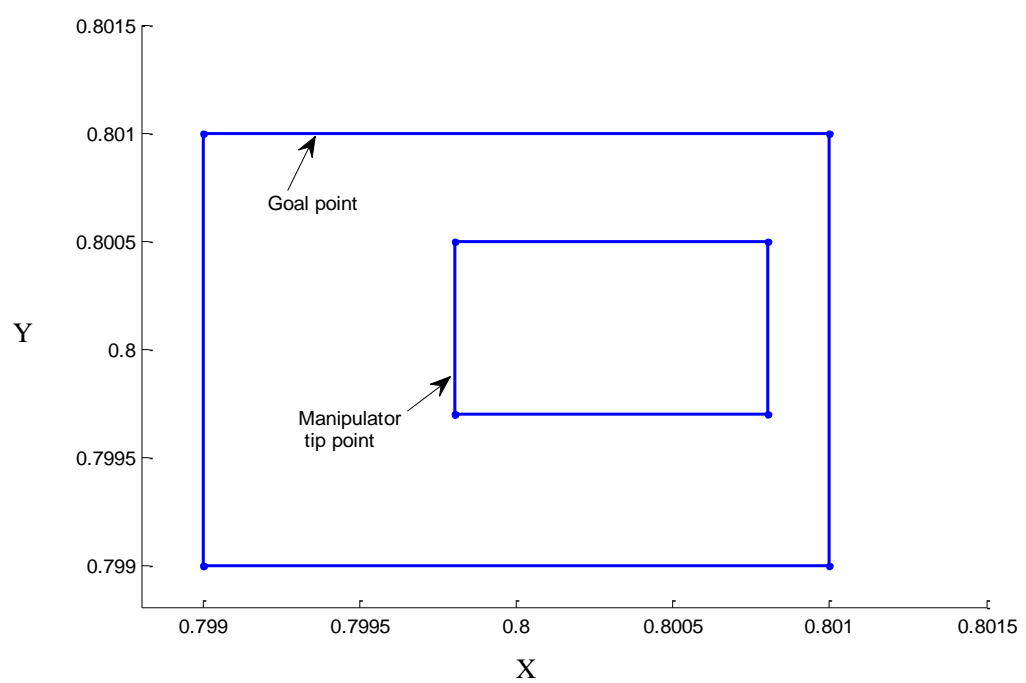

Fig.2. The Output Result - The End-Effector Position Lies Within The Goal Position Precision Range. 
From Fig. 2, it is clear that the manipulator end effector (manipulator tip point) position which is plotted corresponding to the output angles is lie within the goal position.

\section{Conclusion}

Inverse kinematics is an important step in performance metric based design optimization of redundant manipulators. Handling manufacturing tolerances, complex geometry of links, etc. is a concern in design of a manipulator for desired performance accuracy. Interval arithmetic method is a viable technique in dealing with these tolerances, where, point solution is not sought, rather a solution interval is found. This paper attempts to evaluate the inverse kinematics of 3-DOF planar redundant manipulator arm using the interval Newton method. Given a tolerance range and acceptable goal point precision range, this paper develops a procedure to find inverse kinematics solution in intervals for redundant 3-DOF planar manipulator. The inverse kinematics solution simultaneously finds a posture, where, a performance metric is optimized. The performance criterion considered here is the so called manipulability of Jacobian of the manipulator. MATLAB $\odot$ function from the INTLAB toolbox, developed by S.M. Rump, have been utilized in performing the interval numerical computations.

\section{References}

[1] Yoshikawa, T. "Manipulability of robotic mechanisms", The International Journal of Robotics Research, vol. 4, no. 2, pp. 3-9, 1985.

[2] Chirikjian, G. and Burdick, J. "Kinematically optimal hyper-redundant manipulator configurations", IEEE Transactions on Robotics and Automation, vol. 11, pp. 794-806, Dec 1995.

[3] Salisbury, J.K. and Craig, J.J. "Articulated hands: force control and kinematic issues", The International Journal of Robotics Research, Vol. 1, No. 1, pp. 4-17, 1982.

[4] Klein,C. and Blaho, B. "Dexterity measures for the design and control of kinematically redundant manipulators", The International Journal of Robotics Research, Vol. 6, No. 2,72-83, Summer 1987.

[5] Angeles,J. "The design of isotropic manipulator architectures in the presence of redundancies", The International Journal of Robotics Research, Vol. 11, No. 3, 196-201, June 1992.

[6] Rao R.S, Asaithambi A., and Agrawal S.K. "Inverse kinematic solution of robot manipulators using interval analysis", J. of Mechanical Design, 120(1):147-150, 1998.

[7] Castellet, A. and Thomas, F. "Towards an Efficient Interval Method for Solving Inverse Kinematic Problems", International Conference on Robotics and Automation, IEEE, Albuquerque, New Mexico, pp 3615-3620, - April 1997.

[8] Pac M., Rakotondrabe M., Khadraoui S., Popa D., Lutz P. "Guaranteed Manipulator Precision via Interval Analysis of Inverse Kinematics", In IDETC/CIE 2013 August 4-7, 2013, Portland, Oregon, USA.

[9] Klein, C.A. and Huang, C.H. "Review of the Pseudoinverse for control of kinematically redundant manipulators", IEEE Transactions on systems, Man and Cybernetics, VOL. SMC-13, NO. 3, pp 245250, March, 1983.

[10] Mahato, S. K. and Bhunia, A. K. "Interval-Arithmetic-Oriented Interval Computing Technique for Global Optimization”, AMRX Applied Mathematics Research express, Volume 2006, Article ID 69642, Pages $1-19$

[11] Hansen, E. R. and Walster, G. W. "Global Optimization Using Interval Analysis”, Marcel Dekker, New York, NY, 2004.

[12] Hansen, E.R. and Sengupta, S. "Global constrained optimization using interval analysis", in Nickel (1980), pp. 25-47.

[13] Moore, R. E., "Interval Analysis”, Prentice Hall, Englewood Cliffs, NJ, 1966. 
[14] Rump, S. M., 1999. "Intlab interval laboratory", In Developments in Realiable Computing, T. Csendes, edito, Kluwer Academic Publishers, Dordrecht, pp. 77-104.

\section{Author(s) Profiles}

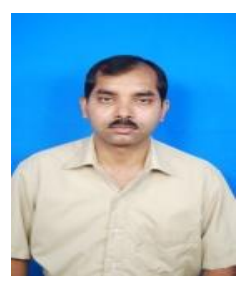

Virendra Kumar received his M.Tech degrees in Mechanical Engineering from N.I.T, Jamshedpur in 2006. Currently, he is working as a Scientist in Robotics and Automation Group, CSIR- Central Mechanical Engineering Research Institute, Durgapur, India. He has published number of research papers in national and international journals, conference proceedings. His current research interests include Robot kinematics and dynamics, optimization, navigation of mobile robot, underwater manipulator.

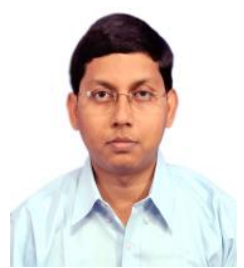

Shibendu Shekhar Roy received his B.E and M.Tech degrees in Mechanical Engineering from R.E. College, Durgapur in 1999 and 2001, respectively. He obtained his Ph.D from IIT, Kharagpur, India, in 2011. Currently, He is an Associate Professor in the Department of Mechanical Engineering, National Institute of Technology, Durgapur, India. He has published a great deal of research papers at national and international journals, conference proceedings, book chapters and filed number of patents in product development. He has written a book on "Modeling and analysis of six-legged robots", which has been published by Lap Lambert Academic Publishing GmbH \& Co. KG, Germany. His current research interests include application of computational intelligent tools like Fuzzy logic, Genetic algorithm, Neural networks etc for modelling of different manufacturing process and robotic systems.

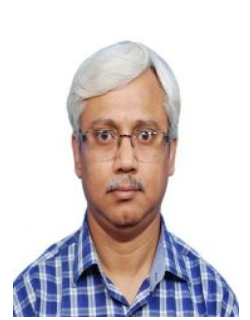

Soumen Sen is working as Principal Scientist at CSIR-Central Mechanical Engineering Research Institute, Durgapur, India since September, 2011. He obtained his Ph.D. in Automation, Robotics, and Bioengineering from the University of Pisa, Italy in 2009. Before his PhD work, Soumen Sen served Department of Atomic Energy, Government of India, from 1994 to 2005 in the Centre for Advanced Technology, Indore and subsequently, in Bhabha Atomic Research Centre, Mumbai. He obtained his Master's degree in Production Technology in 1994 and Bachelor's degree in Mechanical Engineering in 1992. His research activities include variable stiffness/impedance actuation, flexible joint robots, exoskeletons and prostheses, robot design, control and manipulation, underwater manipulation, biomimetic actuators and fin actuation and control.

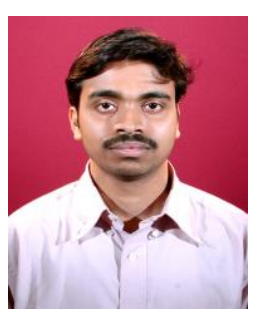

Subhra Kanti Das West Bengal, India, Scientist received the Bachelor of Engineering in Computer Science and Engineering from University of Kalyani, West Bengal, India in 2004. $\mathrm{He}$ is a PhD scholar at department of CSE in University of Jadavpur, West Bengal, India. His research interests include probabilistic measures towards navigation and localization of underwater mobile robotic systems, and application of AI techniques in development of control software architecture. 


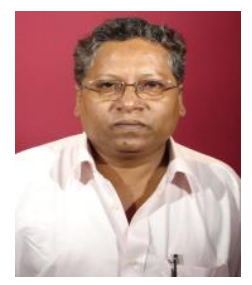

Sankar Nath Shome a 1980 mechanical engineering graduate from Bengal Engineering College (Now IIEST), Shibpur, India received the MTech degree from IIT, Kanpur, India and the $\mathrm{PhD}$ degree in mechanical engineering from National Institute of Technology, Durgapur, India. He is working as a chief scientist at CSIR-CMERI, Durgapur and a professor in the engineering science faculty of AcSIR, India and acting as coordinator and dean of the academic unit at CSIR-CMERI, Durgapur. He initiated various programmes on intelligent robotics and successfully implemented several projects related to robotics. His areas of interest include robotics, mechanisms, control, motion planning and autonomous underwater vehicle technologies. Presently he is involved in the design and development of hybrid modular AUV with cooperative modules.

How to cite this paper: Virendra Kumar, Soumen Sen, S.S. Roy, S.K Das, S.N.Shome,"Inverse Kinematics of Redundant Manipulator using Interval Newton Method", IJEM, vol.5, no.2, pp.19-29, 2015.DOI: 10.5815/ijem.2015.02.03 